\title{
Review \\ Analysis and Comparison of Electromagnetic Microrobotic Platforms for Biomedical Applications
}

\author{
Ruipeng Chen ${ }^{t, \ddagger}(\mathbb{D}$, David Folio $*, \neq(\mathbb{D}$ and Antoine Ferreira $* \mathbb{C}$ \\ INSA Centre Val de Loire, Université d'Orléans, PRISME EA 4229, 45000 Orléans, France; \\ ruipeng.chen@insa-cvl.fr \\ * Correspondence: david.folio@insa-cvl.fr (D.F.); antoine.ferreira@insa-cvl.fr (A.F.); \\ Tel.: +33-(0)2-48-48-40-75 (D.F.); +33-(0)2-48-48-40-79 (A.F.) \\ + Current address: INSA Centre Val de Loire, 88 Boulevard Lahitolle, 18000 Bourges, France. \\ $\ddagger$ These authors contributed equally to this work.
}

Featured Application: Targeted drug delivery using magnetic micro/nanorobots.

check for updates

Citation: Chen, R.; Folio, D.; Ferreira, A. Analysis and Comparison of Electromagnetic Microrobotic Platforms for Biomedical Applications. Appl. Sci. 2022, 12, 456. https://doi.org/10.3390/app12010456 Academic Editors: Irene Fassi and Serena Ruggeri

Received: 23 November 2021 Accepted: 29 December 2021 Published: 4 January 2022

Publisher's Note: MDPI stays neutral with regard to jurisdictional claims in published maps and institutional affiliations.

Copyright: (C) 2022 by the authors. Licensee MDPI, Basel, Switzerland. This article is an open access article distributed under the terms and conditions of the Creative Commons Attribution (CC BY) license (https:// creativecommons.org/licenses/by/ $4.0 /)$.

\begin{abstract}
Magnetic microrobotics is a promising technology for improving minimally invasive surgery (MIS) with the ambition of enhancing patient care and comfort. The potential benefits include limited incisions, less hemorrhaging and postoperative pain, and faster recovery time. To achieve this, a key issue relies on the design of a proper electromagnetic actuation (EMA) setup which is based on the use of magnetic sources. The magnetic field and its gradient generated by the EMA platform is then used to induce magnetic torque and force for microrobot manipulations inside the human body. Like any control systems, the EMA system must be adapted to the given controlled microrobot and customized for the application. With great research efforts on magnetic manipulating of microrobots, the EMA systems are approaching commercial applications, and their configurations are becoming more suitable to be employed in real medical surgeries. However, most of the proposed designs have not followed any specific rule allowing to take into account the biomedical applications constraints. Through reviewing the different proposed EMA systems in the literature, their various specifications and configurations are comprehensively discussed and analyzed. This study focus on EMA platforms that use electromagnets. From this review and based on the biomedical application specifications, the appropriate EMA system can be determined efficiently.
\end{abstract}

Keywords: electromagnetic actuation; medical magnetic microrobots; minimally invasive surgery

\section{Introduction}

Magnetically actuated microrobots are of great interest for the development of innovative biomedical operations. The need to improve interventional operations has led to a wide range of minimally invasive procedures. Since most current operations are limited by the manual action of the surgeon, various robotic systems have been proposed to enhance minimally invasive surgery (MIS) [1-14]. Unlike the need of using rigid instruments with dexterous distal wrists, it is commonly more appropriate to use robotic tools that access internal tissues through small skin incisions [14-19]. Thanks to these medical robotic solutions the acceptance of their uses in clinical practice has been improved. For instance, researchers from the robotics field have developed solutions like robotized tele-echography to provide skilled medical care to isolated patients [20]. Meanwhile, various microrobotic systems have arisen to further reduce trauma, create new diagnosis tools and therapeutic procedures. For example, wireless microrobots with size of less than a millimeter are investigated to navigate within the body for targeted therapies [21-23].

Indeed, the design of miniaturized and versatile microrobotic systems potentially allows access to the entire human body, thus offering localized diagnoses and treatments with more precision and efficiency, but also to consider new procedures. For example, 
wireless microrobots, smaller than a millimeter, can navigate the body to perform targeted therapies [21-23]. A key issue lies in the actuation of such untethered microrobots within the human body. Among the various techniques developed so far, electromagnetic actuation (EMA) is considered to be the most promising one [13,21-50]. To this aim, many EMA platforms have been proposed to control untethered magnetic microrobots for biomedical applications [29,42,48-81]. Using EMA system circumvents the need of embedding power power sources into the microrobot [13,21,22,25,46,82]. Magnetic microrobots can be operated remotely and precisely by controlling external electromagnetic fields. This allows the microrobots to assist the surgeon by increasing the dexterity of the MIS procedure [13,14,22,25,46,57,78,81].

To properly manipulate magnetic microrobots for the realization of reliable given biomedical applications, the EMA platform is one of the key elements. Consequently, the choice, the number and the placement of its magnetic sources are of prime importance [81]. Basically, magnetic sources can be produced by either permanent magnets [29,51-56] or electromagnets [42,48,49,57-78]. Nevertheless, most of the numerous EMA designs proposed by researchers do not follow any specific rule. It is not easy to choose the appropriate solution for a given application. This review aims to summarize and compare the existing EMA systems regarding MIS procedures, focusing on those using electromagnets. At first, the electromagnetic sources of EMA systems could be organized either in a twodimensional (2D) or in a three-dimensional (3D) arrangements, and apply properly to the different parts of the human body, as presented in Figure 1. As illustrated in Figure 1A, the 2D placement of magnets could be useful for surface operations such as angioma or cosmetic treatments. In the meanwhile, most MIS interventions require a 3D workspace, hence, the EMA system should be arranged in 3D above Figure $1 \mathrm{~B}$ or around Figure $1 \mathrm{C}$ the workspace as well.

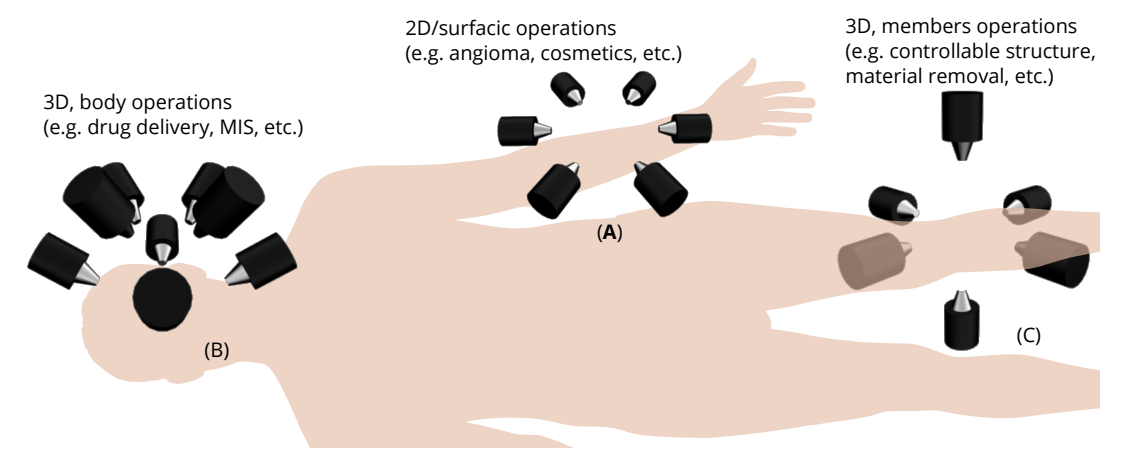

Figure 1. The concept of EMA system applying for various biomedical applications.

\section{Theoretical Background}

\subsection{Magnetic Manipulation}

EMA systems consisting of several electromagnets allow generating a magnetic field and/or a gradient field in a given workspace, as shown in Figure 2. These fields induce a magnetic torque and a force on the magnetized materials of the untethered microrobots. The expression of the magnetic field generated by an electromagnetic coil is derived from a single wire and the magnetic dipole. The magnetic field from any electromagnetic coil $c$ can be approximated as a magnetic dipole characterized by its magnetic moment $\mathbf{m}_{c}$, and the point-dipole model is proposed. It can be shown that the magnetic field and its gradient are proportional to the electric current $i_{c}$ flowing through the coil $c$. The overall magnetic field generated by the $n$-coils is the superposition of each field. The magnetic field and its gradient are then expressed as: 


$$
\begin{aligned}
\mathbf{B}(\mathbf{p}) & =\mathcal{B}(\mathbf{p}) \mathbf{i}=\left(\begin{array}{llll}
\mathcal{B}_{x} & \mathcal{B}_{y} & \mathcal{B}_{z}
\end{array}\right)^{\top} \mathbf{i} \\
\nabla \mathbf{B}(\mathbf{p}) & =\mathcal{G}(\mathbf{p}) \mathbf{i}=\left(\begin{array}{lllll}
\frac{\partial \mathcal{B}_{x}}{\partial x} & \frac{\partial \mathcal{B}_{x}}{\partial y} & \frac{\partial \mathcal{B}_{x}}{\partial z} & \frac{\partial \mathcal{B}_{y}}{\partial y} & \frac{\partial \mathcal{B}_{y}}{\partial z}
\end{array}\right)^{\top} \mathbf{i}
\end{aligned}
$$

where $\mathbf{i}=\left(i_{1}, \ldots, i_{n}\right)^{t}$ is the electric current vector.

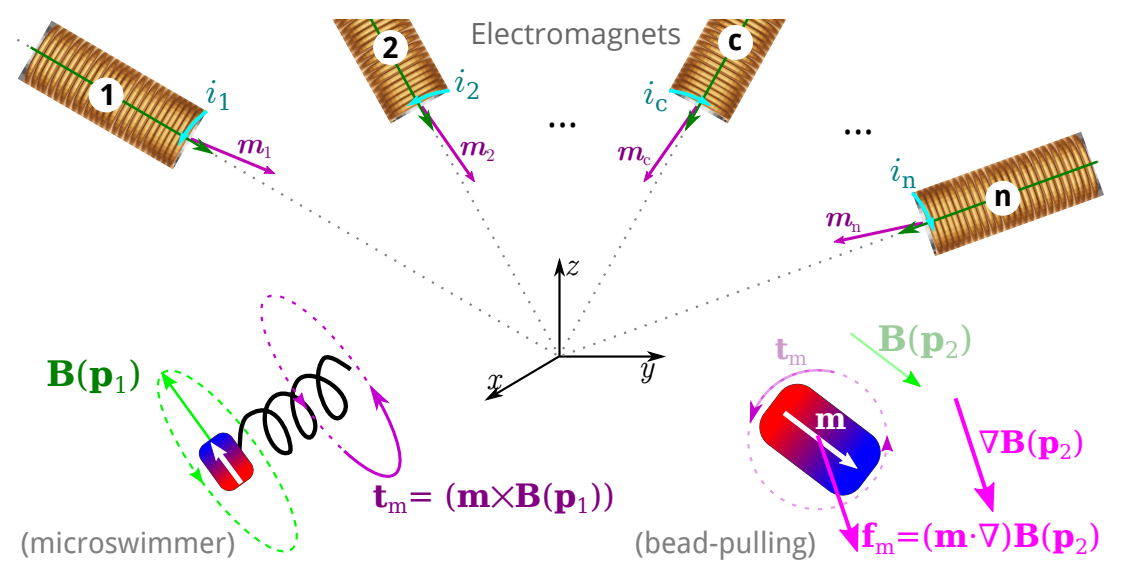

Figure 2. Illustration of the use of the magnetic manipulation of untethered microrobots.

When the magnetic dipole moment and the magnetic field are given, the induced magnetic torque and force can be easily obtained by the Maxwell's equations. The induced magnetic field aligns the microrobot to a desired direction, and the magnetic force provides the propulsion force to move the microrobot to complete the task. Through the mathematical transformation, and for more convenient investigations of the magnetic actuation properties, the equations of torque and force can be rearranged into the following expression:

$$
\left(\begin{array}{c}
\mathbf{t}_{m} \\
\mathbf{f}_{m}
\end{array}\right)=\left(\begin{array}{c}
\mathbf{m} \times \mathcal{B}(\mathbf{p}) \\
\mathbf{m}^{t} \mathcal{G}(\mathbf{p})
\end{array}\right) \mathbf{i}=\mathcal{A}(\mathbf{m}, \mathbf{p}) \mathbf{i}
$$

where $\mathcal{A}(\mathbf{m}, \mathbf{p}) \in \mathbb{R}^{6 \times n}$ is an actuation matrix mapping the current to the applied magnetic wrench. This magnetic actuation matrix is a function of both the position $\mathbf{p} \in \Omega$, and the magnetic moment $\mathbf{m}$ of the microrobot.

Therefore, substituting Equations (1) and (2) into Equation (3), the equations of torque and force can be presented by the actuation matrix $\mathcal{A}(\mathbf{m}, \mathbf{p})$ in the further details as:

$$
\mathcal{A}(\mathbf{m}, \mathbf{p})(\mathbf{i})=\left(\begin{array}{cccccccc}
m_{x} & m_{y} & m_{z} & 0 & 0 & 0 & 0 & 0 \\
0 & m_{x} & 0 & m_{y} & m_{z} & 0 & 0 & 0 \\
-m_{z} & 0 & m_{x} & -m_{z} & m_{y} & 0 & 0 & 0 \\
0 & 0 & 0 & 0 & 0 & 0 & -m_{z} & m_{y} \\
0 & 0 & 0 & 0 & 0 & m_{z} & 0 & -m_{x} \\
0 & 0 & 0 & 0 & 0 & -m_{y} & m_{x} & 0
\end{array}\right)\left(\begin{array}{c}
\frac{\partial \mathcal{B}_{x}}{\partial x} \\
\frac{\partial \mathcal{B}_{x}}{\partial y} \\
\frac{\partial \mathcal{B}_{x}}{\partial z} \\
\frac{\partial \mathcal{B}_{y}}{\partial y} \\
\frac{\partial \mathcal{B}_{y}}{\partial z} \\
\mathcal{B}_{x} \\
\mathcal{B}_{y} \\
\mathcal{B}_{z}
\end{array}\right)(\mathbf{i}
$$

Each column of the matrix $\mathcal{A}(\mathbf{m}, \mathbf{p})$ represents the wrench on the force and torque per unit current created by each electromagnet. If there are greater than $n>6$ electromagnets, the actuation matrix $\mathcal{A}(\mathbf{m}, \mathbf{p})$ leads to a better conditioned matrix, a more 
isotropic workspace $\Omega$, a reduction of singularity configurations, and lower current requirements $[72,73,81]$. In such cases, $n>6$, the EMA system is said "redundant" for the task. Especially, if $\mathcal{A}(\mathbf{m}, \mathbf{p})$ has a full rank, for a desired force, $\mathbf{f}_{m}^{\star}$ and torque, $\mathbf{t}_{m}^{\star}$, the actuation currents $\mathbf{i}$ can be calculated from the pseudo-inverse:

$$
\mathbf{i}=\mathcal{A}^{+}(\mathbf{m}, \mathbf{p})\left(\begin{array}{c}
\mathbf{t}_{m}^{\star} \\
\mathbf{f}_{m}^{\star}
\end{array}\right)
$$

If $n<6$, the pseudo-inverse would be a least-squares approximation. Hence, for a controlled force and torque, the input current can be obtained only if the pseudo-inverse of $\mathcal{A}(\mathbf{m}, \mathbf{p})$ exists. This derivation on the controlled current $\mathbf{i}$ can be similarly extended for controllers that require torque and/or force control [57].

\subsection{Manipulation Analysis}

From the mathematical analysis, the rank of force equation is 3 and the rank of torque equation is 2 , the microrobot can maximally achieve three degrees-of-freedom (DOFs) in translation and two DOFs in rotation. Next, to achieve the five DOFs control of the microrobot, the minimum number of electromagnets is mathematical estimated. The three electromagnets can be used for three DOFs force control at a point, but normally five electromagnets are required when the orientation of the microrobot is dynamic changed. The number of electromagnets can be reduced to four, but either a nonmagnetic restoring torque or a nonmagnetic restoring force is required to stabilize the system. For two DOFs torque control, only three electromagnets are required because the three coils can generate a 3D field in a workspace. Thus, combined torque and force control requires a minimum of $n=7$ stationary electromagnets. Similarly, the seven electromagnets also need some additional external conditions. To stabilize the five DOFs control of the microrobot, the eight electromagnets are suggested for the fixed configuration system [83]. Reconfigurable EMA system can achieve similar control authority to stationary system with fewer electromagnets. Only $n=5$ electromagnets are required for torque and force control. Therefore, the mobile electromagnets are more particularly considered for the biomedical applications. Indeed, the field shape in the workspace can be modified by changing the location or orientation of the electromagnets during the magnetic actuation of the microrobot [81].

\subsection{Discussions}

Various arrangements of electromagnetic coils can generate various magnetic field distributions. The EMA setup should be properly defined with respect to the envisioned biomedical application. To do so, the main characteristics should be specified, such as: the environment of the workspace, the type of microrobot and the various magnetic tasks. The required number of electromagnets for different motions control has been studied in past works $[45,81,83]$. On this basis, the relations between the specifications and the number of coils to design an EMA system can be proposed, and are depicted in Figure 3.

Specifically, for the choice of a proper EMA system, five main characteristics of an application are required:

1. The dimensions of workspace;

2. The media of the environment;

3. The type of microrobots;

4. The medical tasks;

5. The required motion control.

First of all, the dimension of workspace is determined by the desired biomedical application that can be either 2D or 3D. The media of workspace could be divided into easy-to-operate and non-easy-to-operate for the placed microrobot. Commonly, the media with high viscosity or non-Newtonian fluid and the flowing status are difficult conditions to manipulate microrobot. In contrast, low viscosity and static environment are easy for the operation of microrobot. 
Moreover, the type of applied microrobot and its locomotion must be specified. Especially, the helical microrobot or microswimmer could be selected to move in a flowing environment and/or high viscosity media since these microrobots can perform drilling motion by the rotating magnetic field (see also Figure 2). Combining with magnetic force produced by magnetic gradient, the helical microrobot or microswimmer can be also actuated by a strong propulsion force. Besides, the cylindrical, ellipsoidal, spheroidal and irregularly shaped microrobot are chosen to the suitable environments. In addition, the type of locomotion of the microrobot should be determined with respect to the given application. As presented in Figure 3, six main types of biomedical applications are here considered. However, a distinction is made according to a 2D or 3D workspace that is considered. For a 2D workspace, as illustrated in Figure 3a, four main types of biomedical applications are considered for a 2D workspace: (i) surface treatment, (ii) marking/sensing, (iii) in vitro micromanipulation, and (iv) controllable structure. Whereas for a 3D biomedical operations, the main types of tasks are: (i) material removal, (ii) marking/sensing, (iii) targeted therapy, and (iv) controllable structure, as depicted in Figure 3b. For instance, almost all types of microrobot could be used for targeted drug delivery. However, the spheroidal microrobot rather than the helical microrobot is suitable for marking/sensing application. If a helical microrobot is applied for targeted drug delivery, the possible motion of microrobot is required as translation and rotation. If a spheroidal microrobot is used for targeted drug delivery, the possible motion could be translation, rotation and punching.
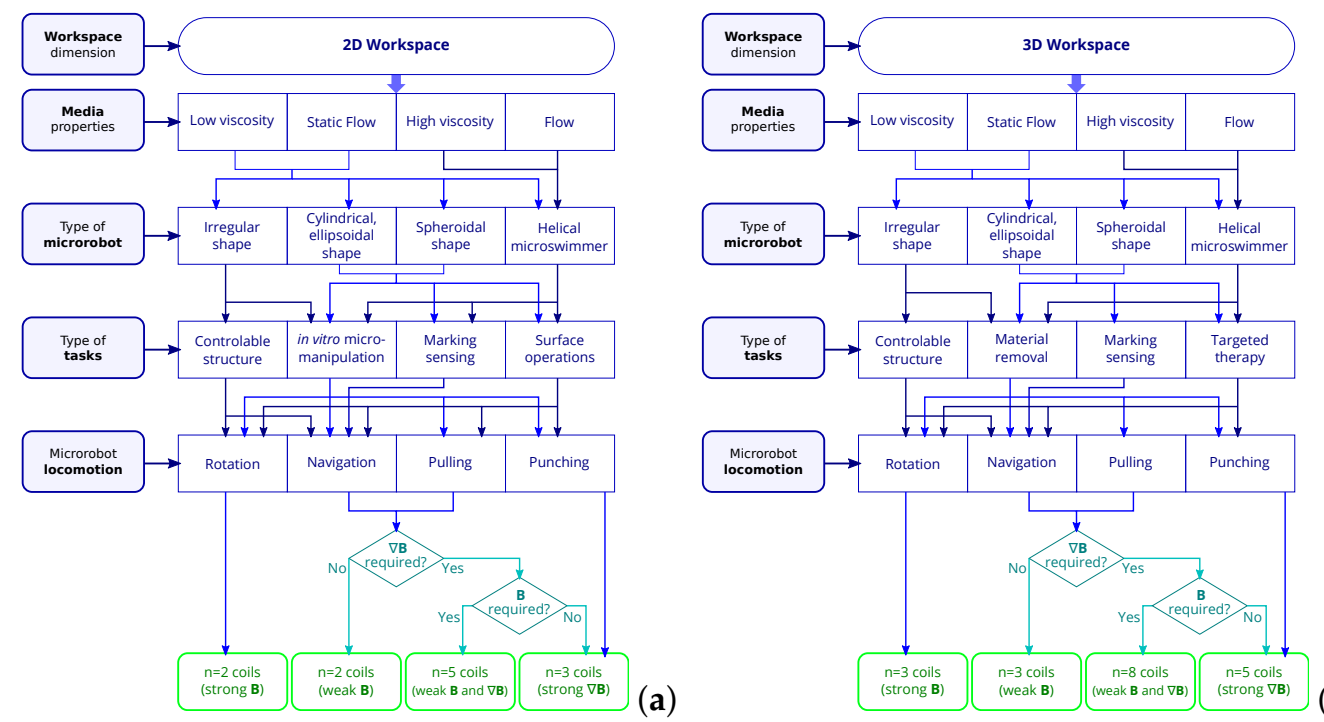

Figure 3. The diagram of the specifications of EMA system design for (a) 2D and (b) 3D workspace.

Finally, the number of electromagnetic coils is determined by the specific motions of the selected microrobot. For instance, the translational locomotion can be achieved by the magnetic force on the spheroidal microrobot, and it can also be reached by the magnetic torque generated by rotating magnetic field on the helical microrobot. Considering a 3D workspace as an example, the reliable locomotion of spheroidal microrobot requires $n=5$ numbers of electromagnets, while the locomotion of a helical microrobot only demands $n=3$ numbers of electromagnets. Similarly, other necessary information for designing EMA system can also be obtained in the same way following Figure 3. Therefore, from the above five requirements, the specifications of desired EMA system are obtained.

\section{The Electromagnetic Microrobotic Systems}

The magnetic microrobot can be efficiently actuated by the utilization of magnetic field and/or its gradient. This magnetic field could be generated from an EMA platform, that must obviously comprises some electromagnetic sources. The magnetic sources could be produced by either permanent magnets [29,51-56] or electromagnets $[42,48,49,57-78]$, that 
should be selected according to the specified biomedical application. The main advantage of permanent magnet sources is that they do not require an external power supply, and they exhibit an advantageous volume to field-strength ratio [52]. However, in such case the magnetic fields can not be accurately adjusted or switched off [54]. In contrast, electromagnets can generate appropriate and flexible magnetic fields to effectively control the movement of microrobots. This study focuses on applications where magnetic fields and/or their gradients need to be continuously changed, and EMA setups using electromagnets are primarily considered in the following. The simplest electromagnet is wrapped around an air-filled core. In such case, the magnetic fields or their gradients can be uniformly defined in the workspace, and linear relationship can be expressed with their input currents. However, in such case, the strength of the magnetic field is weaker than using a permanent magnet on an equivalent volume. To increase the strength, a magnetic core with a high magnetic permeability can be added inside the coil to confine and guide the magnetic fields. The magnetic field is related to the electric current as well as to the properties of the magnetic core. Nevertheless, EMA system with several core-filled electromagnets may exhibit nonlinear and coupled behavior.

Furthermore, the EMA systems can also be distinguished into stationary and mobile. Stationary magnetic sources commonly use Helmholtz, Maxwell and saddle coils [61-65,67,69,71,74,84], as in MRI system [58,85-87], to induce magnetic fields and gradients that only are controlled by the current flowing into the electromagnetic coils. With such stationary configurations, the magnetic manipulation of the microrobot together with the workspace geometry remains limited by the stationary arrangement of coils. Conversely, moving magnets (e.g., actuated by a robotic system) can move around the target to enhance the manipulability of microrobots [88,89]. As the magnetic sources usually remain close to the microrobot, the moving coils also reduce the energy demand. In addition, they can change the local field distribution by adjusting the positions and/or orientations of the magnets $[60,73]$.

As shown in Figure 4a, Fountain et al. [51] propose the use of nonuniform magnetic fields emanated from from a single rotating-permanent magnet manipulator for the control of magnetic helical microrobots, where the robotic arm brings the magnet closer to the patient, and the axial and radial controls cause the local magnetic field to change. Stereotaxis Inc. (Stereotaxis Inc., St. Louis, MO, USA, http:/ / www.stereotaxis.com accessed on: 23 November 2021 has developed and commercialized the Niobe ${ }^{\circledR}$ robotic magnetic navigation system presented in Figure $4 \mathrm{~b}$. Niobe ${ }^{\circledR}$ uses two permanent magnets mounted on pivoting arms and positioned on opposing sides of the operating table to control proprietary catheters and guide-wires that have very small magnets at their distal tips. To circumvent the uncontrollability of the magnetic field generated by permanent magnets, Véron et al. [88] investigate a robot-assisted magnetic manipulation system with several mobile electromagnetic coils as represented in Figure 5b, where a robotic system keeps full dexterity for the use of electromagnetic coils while reducing energy consumption by a nearer manipulation. Furthermore, Yang et al. [89] demonstrate an electromagnetic manipulation system with three parallel mobile coils named DeltaMag and represented in Figure 5b. The proposed EMA system can remotely control the magnetic untethered devices in an enlarged workspace, moreover, the electromagnetic coils are actuated through the parallel mechanism to achieve the flexibility of their placement. Thus, the DeltaMag system proves that the mobile sources generated by moving electromagnets can improve the manipulability of localization to close the vicinity of the desired area and bring the good space utilization. 


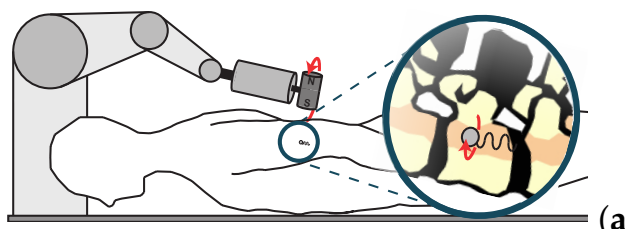

(a)

(b)

Figure 4. Examples of EMA systems with moving permanent magnets: (a) conceptual image of a rotating-permanent-magnet manipulator proposed by Fountain et al. [51], and (b) the Stereotaxis Niobe ${ }^{\circledR}$ consisting of two robotically-controlled magnets next to the table.

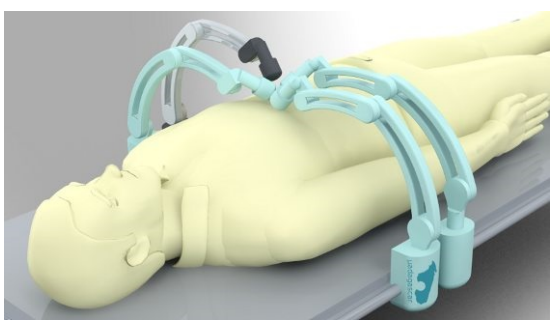

(a)

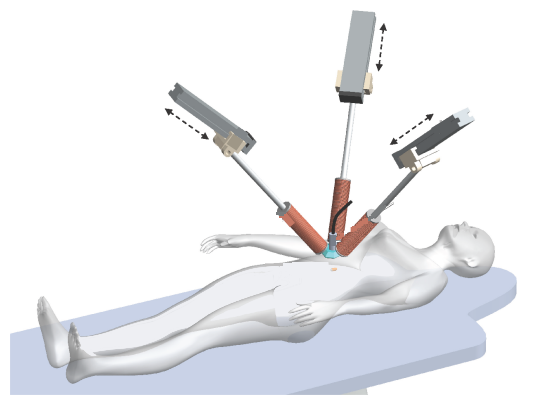

(b)

Figure 5. Examples of EMA systems with moving electromagnets: (a) the robot-assisted magnetic manipulation proposed by Véron et al. [88], and (b) the DeltaMag system consisting of three parallel mobile coils [89].

Here, we present a brief overview of different EMA setups in the following paragraphs. These electromagnetic microrobotic platforms can be divided into two-dimensional and three-dimensional manipulations. The status of electromagnetic coils could be stationary or mobile. The functions of designed electromagnetic platform vary according to the configuration of electromagnets. The Helmholtz coils pair, Maxwell coils pair, uniform saddle coils pair and gradient saddle coils pair are basic electromagnets configurations as shown in Figure 6 and are commonly used to generate a uniform magnetic field or gradient in a given workspace. The magnetic field intensity $H_{h}, H_{m}, H_{u}$ and $H_{g}$ of them can be computed as follows, respectively:

$$
\begin{aligned}
& H_{h}=\left(\begin{array}{lll}
d_{h} x & 0 & 0
\end{array}\right)^{\top} \\
& d_{h}=\left(\frac{4}{5}\right)^{\frac{3}{2}} \frac{i_{h}}{r_{h}} \\
& H_{m}=\left(\begin{array}{lll}
g_{m} x & -0.5 g_{m} y & -0.5 g_{m} z
\end{array}\right)^{\top} \\
& g_{m}=\frac{16}{3}\left(\frac{3}{7}\right)^{\frac{5}{2}} \frac{i_{m}}{r_{m}^{2}} \\
& H_{u}=\left(0, d_{u}, 0\right)^{\top} \\
& d_{u}=0.6004 \frac{i_{u}}{r_{u}} \\
& H_{g}=\left(\begin{array}{lll}
g_{g} x & -2.4398 g_{g} y & 1.4398 g_{g} z
\end{array}\right)^{\top} \\
& g_{g}=\cos ^{-1}\left(1-\frac{3}{2 a^{2}} \frac{16}{3 \pi}\left(\frac{3}{7}\right)^{\frac{5}{2}} \frac{i_{g}}{r_{g}^{2}}\right)
\end{aligned}
$$

where $i_{h}$ and $r_{h}=r$ are the current and the radius of the Helmholtz coils; $i_{m}$ and $r_{m}=r$ are the current and the radius of the Maxwell coils; $i_{u}$ and $r_{u}=r$ are the current and the radius of the uniform saddle coils; and $i_{g}$ and $r_{u}=g$ are the current and the radius of the gradient saddle coils. 


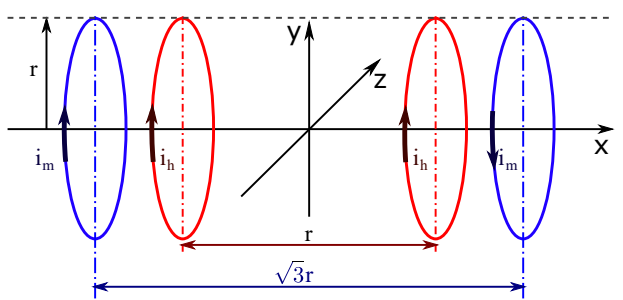

(a)

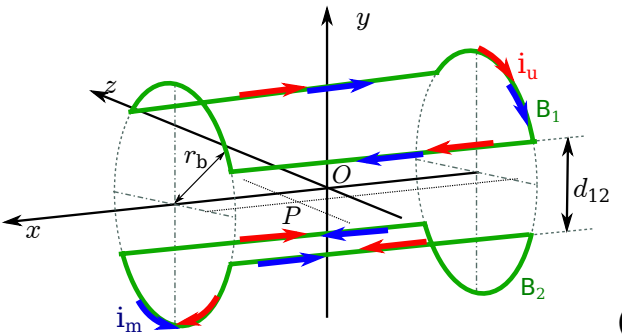

(b)

Figure 6. Basic electromagnets configurations: (a) representation of an Helmholtz (inner red) and Maxwell (outer blue) coils pair.; and (b) representation of saddle-shaped coils: with same current $i_{u}$ flowing in the uniform saddle coil, and current $i_{g}$ in phase opposite for the gradient saddle coil.

As shown in Figure 6a, the Helmholtz set includes two solenoids with same radius $r_{h}$ separated by the distance: $l=r_{h}$, and the Maxwell coil consists of a pair of same coils of radius $r_{m}$ separated with a distance $l=\sqrt{3} r_{m}$. The currents flowing in an Helmholtz coil pair have same intensity and phase, that is: $i_{h}=i_{h \text { left }}=i_{h \text { right }}$, while the currents of Maxwell coils are flowing in opposite phases, that is: $i_{m \text { left }}=-i_{m \text { right }}$ and $i_{m}=\left|i_{m \text { left }}\right|=\left|i_{m \text { right }}\right|$. It is clear that the magnetic fields generated by the combination of a Helmholtz coils pair and a Maxwell coils pair are different from that produced by two Helmholtz coils pairs. Hence, the different configurations of platforms composed of different coils pairs will be investigated. To make it easier to name each magnetic platform, we introduce the abbreviation to identify them with the nomenclature provided in Table 1.

Table 1. ElectroMagnetic Actuation system nomenclature.

\begin{tabular}{clcl}
\hline Symbol & Description & Symbol & Description \\
\hline $\mathrm{H}$ & Helmholtz coils & $\mathrm{M}$ & Maxwell coils \\
$\mathrm{U}$ & Uniform saddle coils & $\mathrm{G}$ & Gradient saddle coils \\
$\mathrm{E}$ & Electromagnet & $\mathrm{C}$ & single coil pair \\
2D & two-dimensions & 3D & three-dimensions \\
$\mathrm{r}$ & rotational & & \\
\hline
\end{tabular}

\subsection{Two-Dimensional Manipulation}

\subsubsection{Platform HMr}

Yesin et al. [49] propose a simple EMA system consisting with one Helmholtz and one Maxwell coils pairs. The coils sets are designed to surround the same rotating axis, as shown in Figure 7. Figure 7a illustrates the schematic diagram of the proposed EMA system. The rotating Helmholtz coils allow aligning the magnetic moment of the microrobot to the desired direction. Maxwell coils are used to directly translate the microrobot in the aligned direction. Thereby the microrobot is actuated in a $2 \mathrm{D}$ space thanks to the manipulation plane obtained by rotating a straight line around the axis of rotation.

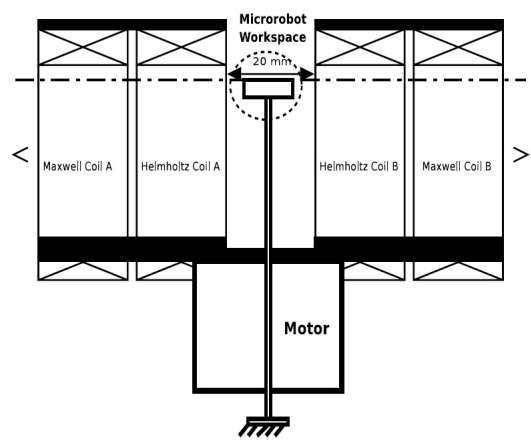

(a)

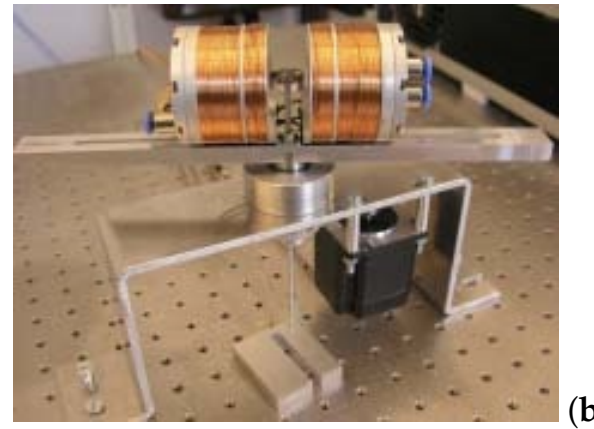

(b)

Figure 7. HMr EMA platform developed by Yesin et al. [49]: (a) scheme of the concept; and (b) a photograph of the magnetic steering system. 


\subsubsection{Platform $2 \mathrm{C}$}

Go et al. [70] propose an EMA system comprising of two stationary pairs of electromagnetic coils to steer microrobot within a 2D plane, and is depicted in Figure 8a. The microrobot can be aligned and steered to a desired location by controlling the currents flowing through each electromagnetic coil. With such simple configuration, the proposed EMA system requires the less power consumption and a smaller volume. To actuate the microrobot, the proposed system needs specific control algorithm of the currents through their coils. Thus, the authors demonstrate real time positioning through the magnetic field control of a calibrate field at the edge of the workspace. This method allows the microrobot to move accurately along desired paths with less positioning errors.
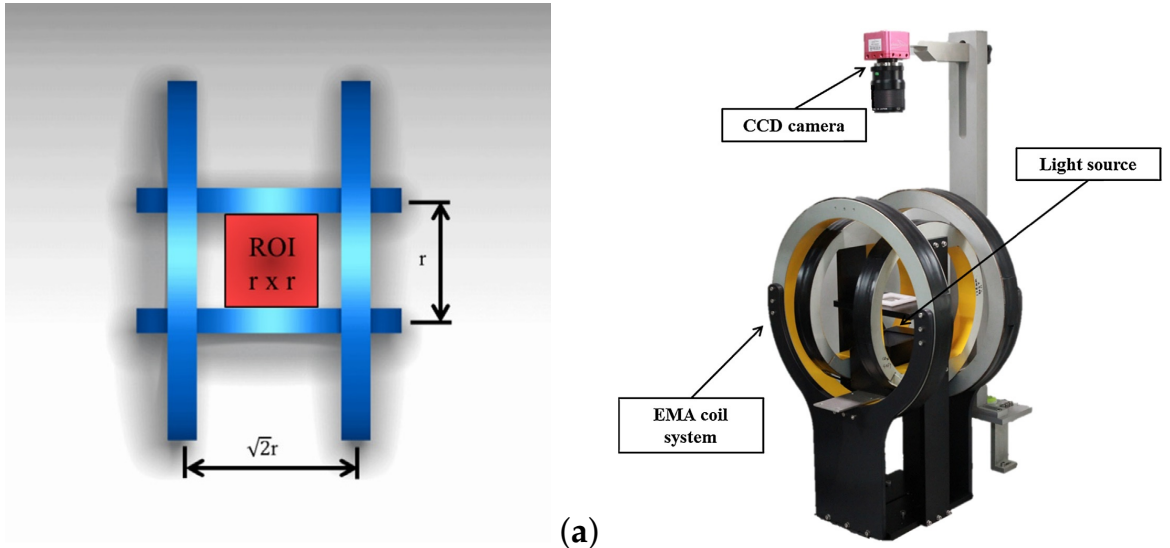

(b)

Figure 8. 2C EMA platform developed by Park et al. [70]: (a) scheme of the concept; and (b) a photograph of the built system.

\subsubsection{Platform 2H2M}

Choi et al. [65] also use Helmholtz and Maxwell coils. The difference is that they are designed by using four sets of fixed coils, as represented in Figure 9. Two Helmholtz coils are placed perpendicular to each other in the $x y$-plane. By adjusting the current ratio between the two pairs of Helmholtz coils, a steerable uniform magnetic field can be generated to drive the magnetic moment of microrobots. Two Maxwell coils are also placed perpendicular to each other in the $x y$-plane. By applying different current ratios between the two pairs of Maxwell coils, the direction of the magnetic force can be adjusted and set.
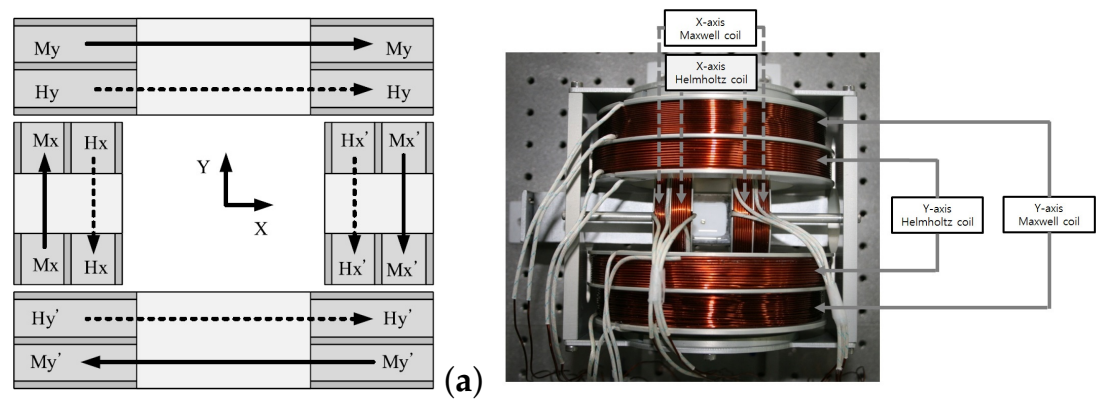

(b)

Figure 9. 2H2M EMA platform developed by Choi et al. [65]: (a) scheme of the concept; and (b) a photograph of the built system.

\subsubsection{Platform HMUG}

Jeon et al. [62] have developed a stationary EMA system composed of one pair of Helmholtz and Maxwell coils, and one pair of uniform and gradient saddle coils, as represented in Figure 10. The proposed EMA system is capable of controlling the 2D locomotion of magnetic microrobot. The geometric shape of saddle coils brings the convenience for manipulation, and especially allows increasing the accessibility to the workspace. Authors 
point out that the designed system is geometrically compact to allow a patient to lie down and magnetically efficient compared with the conventional configuration which has two pairs of Maxwell and Helmholtz coils.

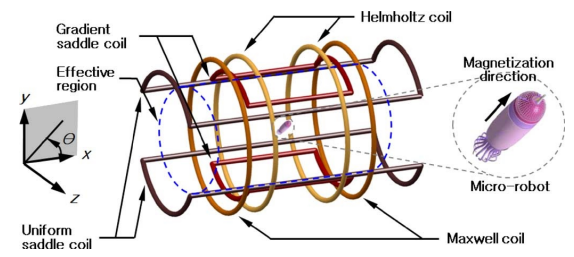

(a)
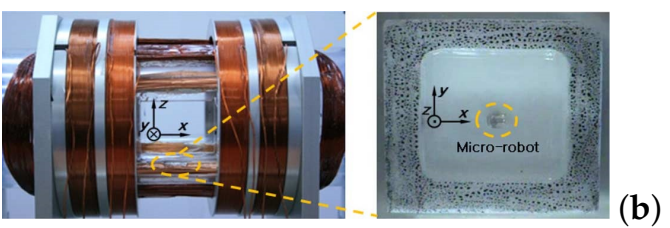

Figure 10. HMUG EMA platform developed by Jeon et al. [62]: (a) scheme of the concept; and (b) a photograph of the built system.

In the system, the desired uniform magnetic field is produced by the pairs of Helmholtz and uniform saddle coils to generate the magnetic torque aligning the orientation of microrobot in the a 2D plane, and the magnetic gradient is induced by the pairs of Maxwell and gradient saddle coils to steer the magnetic microrobot along the given direction. Therefore, this EMA system is able to provide a 2D pointing and positioning control of the microrobot.

\subsubsection{Platform 3H2M}

Go et al. [90,91] have investigated an EMA system with three stationary pairs of Helmholtz coils on each direction, and two stationary pairs of Maxwell coils fixed on $x y$ plane as shown in Figure 11.

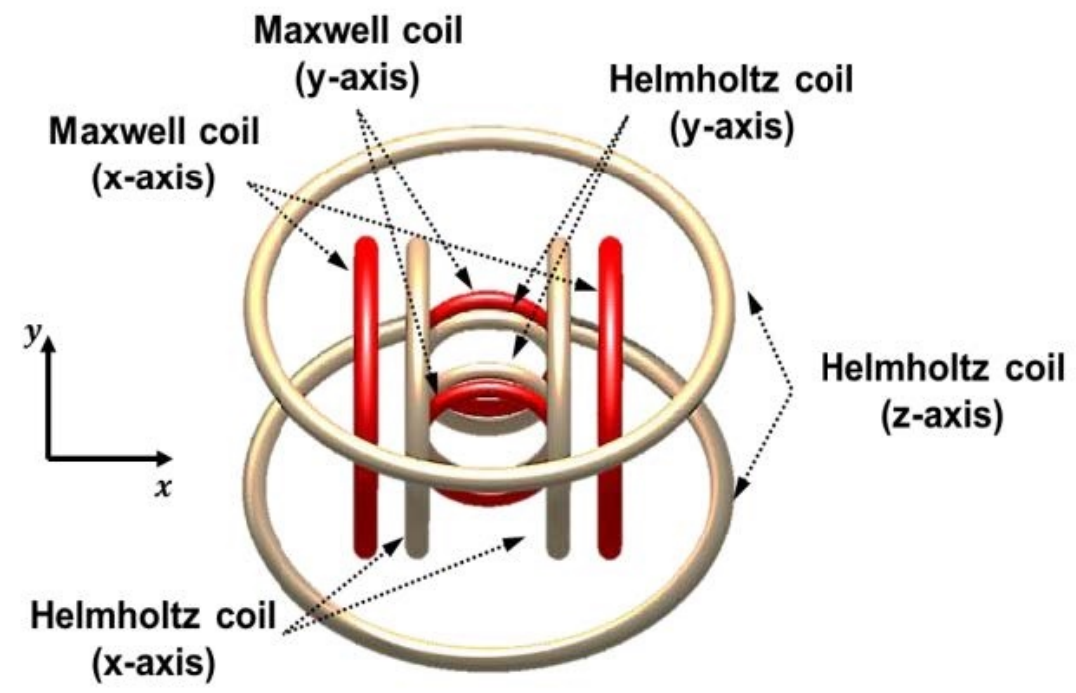

Figure 11. Schematic configuration of EMA system coils developed proposed by Park et al. [90].

\subsubsection{Platform $6 \mathrm{E}$}

Diller et al. [92] have reported a method to control multiple untethered magnetic microrobots independently using an EMA system consisting of six independent air-core electromagnetic coils as shown in Figure 12. Actuated by the applied external magnetic field, multiple microrobots are steered to arbitrary goal positions, while the limited path is also achievable. Hence, the proposed EMA system has the potential to control independently the locomotion of multiple microrobots for different manipulation tasks. 


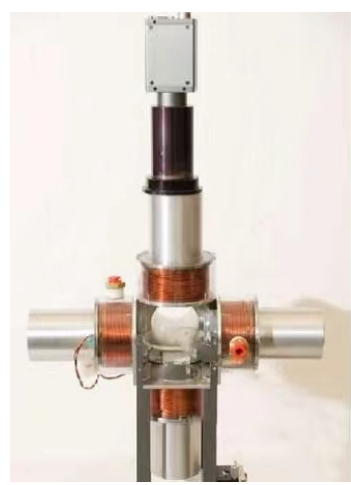

Figure 12. Electromagnetic coil setup consisting of six coils. Two of four horizontal coils are removed to allow viewing of workspace [92].

\subsection{Three-Dimensional Manipulation}

\subsubsection{Platform $3 \mathrm{DMH}$}

In [74], authors present an EMA system composed of three sets of electromagnets, as shown in Figure 13a. The three pairs of coils are placed perpendicularly to generate the magnetic field in the three directions of Cartesian coordinate system. Moreover, the three sets of coils can also provide propulsion force to move the microrobot by the proper control of their currents. Hence, by the combination of Helmholtz and Maxwell coils in each set, the proposed EMA system can provide a simple approach for the 3D actuation of the microrobot in low fabrication cost, that is represented in Figure 13b. The authors report that this EMA platform has the capability of generating the necessary magnetic field and gradient to perform the suitable control of a microrobot on a defined trajectory [74].

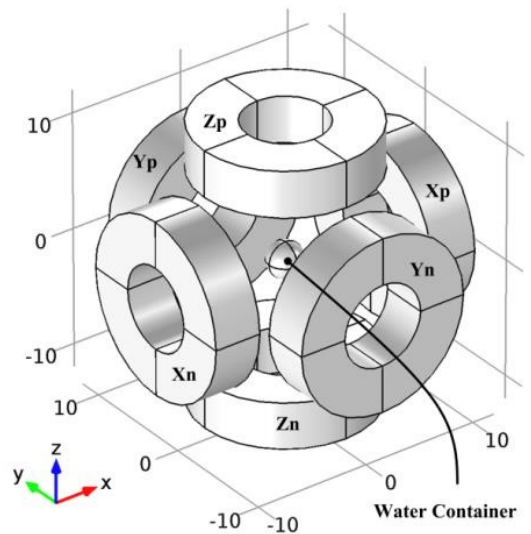

(a)

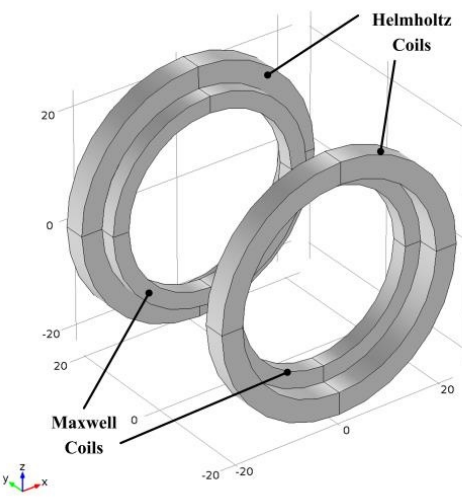

(b)

Figure 13. 3DMH EMA platform developed by Dadkhah et al. [74]: (a) representation of the concept; and $(\mathbf{b})$ the design of a set of electromagnets for a given axis that combines a pair of Helmholtz and Maxwell coils.

\subsubsection{Platform $2 \mathrm{H} 2 \mathrm{Mr}$}

It is also possible to simply obtain the 3D motion control by moving the stationary 2D manipulation plane. For instance, Jeong et al. [61] propose an EMA system for the 3D locomotion of intravascular microrobots by the controlling the rotational plane. The considered system is composed of a stationary Helmholtz-Maxwell coil pairs and a rotational Helmholtz-Maxwell coil pairs, as shown in Figure 14. Two pairs of the Helmholtz coils align the microrobot to the desired direction, and two pairs of the Maxwell coils steer the microrobot on the aligned direction. Figure 14a shows the stationary Helmholtz-Maxwell coils located in the $x$-axis, and the other rotational Helmholtz-Maxwell coils that are set along the $x$-axis. Thus, in the workspace, when a microrobot is not aligned in the desired direction, the two pair of Helmholtz coils generate a uniform magnetic field to align the microrobot in the desired orientation. The uniform magnetic gradient generated by the 
two pairs of Maxwell coils induces the propulsion force to propel the microrobot. As shown in Figure 14a, the rotational Maxwell coil pairs behave similarly to the rotational Helmholtz coil pairs. Hence, the microrobot can be rotated and translated in the same manipulation plane.

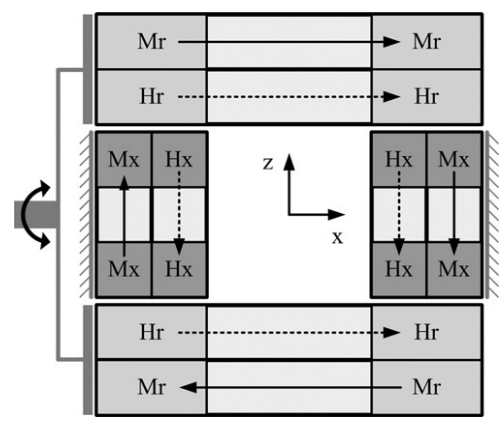

(a)

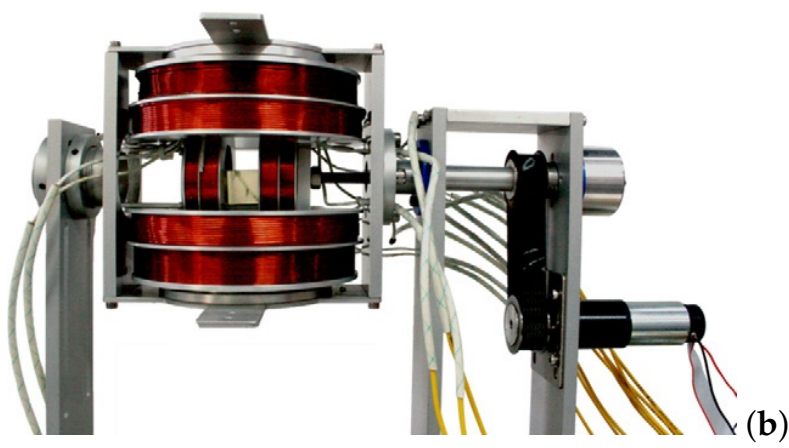

Figure 14. 2H2Mr EMA platform developed by Jeong et al. [61]: (a) scheme of the concept; and (b) a photograph of the built system.

Through the microrobot is actuated in a 2D plane, the a 2D manipulation plane can be expanded to a 3D space by rotating determined plane by rotational coils pairs revolving about the $x$-axis. Therefore, the considered EMA system can achieve a 3D manipulation.

\subsubsection{Platform HMUGr}

Generally, the EMA system constructed from Helmholtz and Maxwell coils takes up a large space. In order to get a smaller volume of the platform and the less consumption of driving energy, in $[62,67]$ the authors have developed an EMA system based on uniform and gradient saddle coils for the 3D locomotion of a microrobot. Referring to the structure given in [67], the pair of Maxwell-Helmholtz coils and the pair of the uniform-gradient saddle coils are perpendicularly fixed (see also Figure 15). Such arranged configuration can realize the $2 \mathrm{D}$ locomotion of a microrobot.
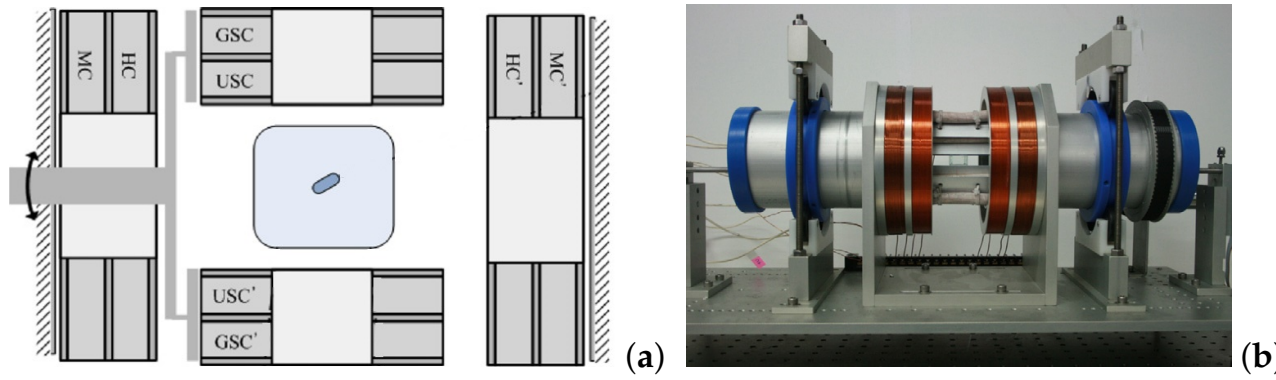

Figure 15. HMUGr EMA platform developed by Choi et al. [67]: (a) scheme of the concept; and (b) a photograph of the built system.

Similarly, the designed EMA system expands the 2D to the 3D actuation by rotating saddle coil pairs. With such structure of rotating saddle coils, the considered system actually provides a more flexible and accessible workspace. In the Figure 15a, the 2D control of orientation of microrobot is realized by using one pair of Helmholtz coils fixed along $x$-axis, and one pair of uniform saddle coils placed around the $x$-axis. Once the microrobot is aligned along the desired angle, the pairs of Maxwell and gradient saddle coils will produce the necessary propulsion force owing to the induced magnetic gradient.

It is noted in $[62,67,93]$ that the systems with the structure of saddle coil allow the less power consumption with a more compact setup. Moreover, such structures are suitable for the actuation of the medical microrobot in vivo. 


\subsubsection{Platform H2US-MUG}

Jeon et al. [63] have proposed an EMA system composed of one pair of Helmholtz coils, two pairs of uniform saddle coils, one pair of Maxwell coils and one pair of gradient saddle coils, that is illustrated in Figure 16. The Maxwell and gradient saddle coils pairs are nested into other coils. Authors demonstrate that their EMA system can perform helical and translational motions of a microrobot in several organs of the human body such as the central nervous system, the urinary system, the eye and blood vessel. The considered EMA setup is able to generate rotating magnetic fields thanks to the Helmholtz and uniform saddle coils for the sufficiently precise corkscrew motions of the helical microrobot; and can also provide uniform magnetic gradient field for the transverse movement of the microrobot along the axial direction via the Maxwell and gradient saddle coils pairs, which can apply for several organs of human body such as the urinary system, the eye or the nervous system.

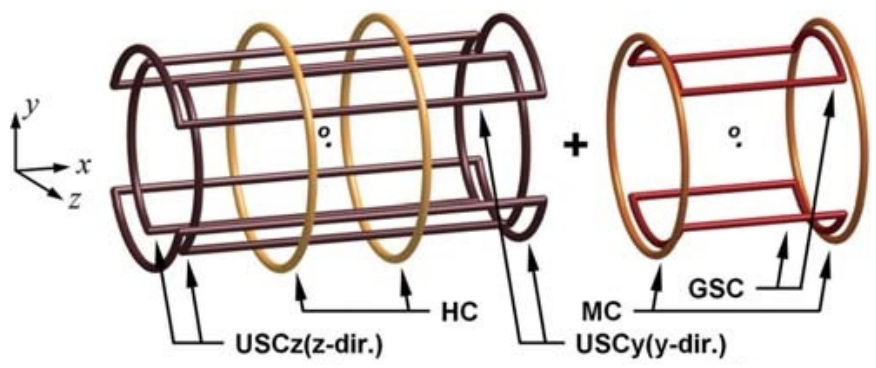

Figure 16. Schematic diagram of the H2US-MUG EMA system developed by Jeon et al. [63].

\subsubsection{Platform $3 \mathrm{DH}$}

Mahoney et al. [94] have developed an EMA system consisting of three nested Helmholtz coil pairs, as illustrated in Figure 17. The three pairs of Helmholtz coils are arranged perpendicularly to allow generating an uniform magnetic field whose orientation can be controlled in the 3D workspace. The authors have also proposed an open-loop algorithm for the velocity control with gravity compensation for the helical microrobot. To steer the microswimmer, the 3D rotating magnetic field is generated by each pair of Helmholtz coils in the workspace. Assuming that the microswimmer always rotates in synchronization with the applied magnetic field, the central axis of the microswimmer is asymptotically converging on the rotation axis of the magnetic field. The desired velocity of the microrobot is provided with a precalculated frequency of the rotating magnetic field. Thus, such EMA configuration can be used to actuate remotely the medical microrobot for performing tasks, such as targeted therapy, tissue removal and remote sensing, by controlling its orientation and rotation speed intuitively, especially in the low-Reynolds-number environment.

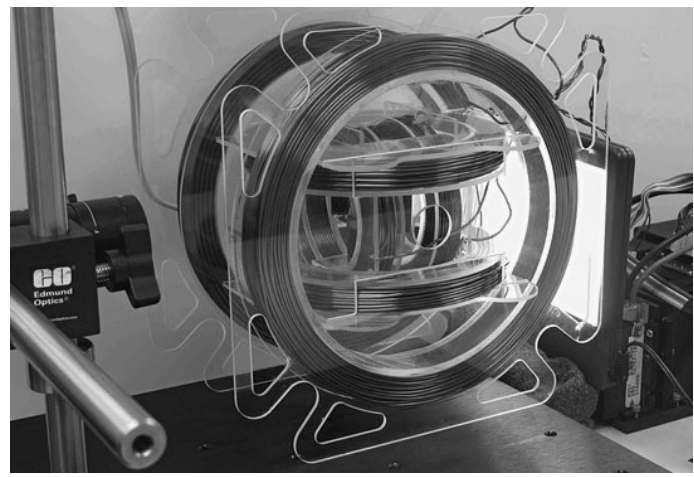

Figure 17. Photograph of the 3DH EMA setup developed by Mahoney et al. [94]. 


\subsubsection{Platform $8 \mathrm{E}$}

To achieve the 3D independent control for multiple microrobots, an EMA system including eight iron-core electromagnetic coils has been developed [95]. Figure 18a illustrates the proposed setup, where the iron-core electromagnets are all pointing to the common workspace center. The 3D motions of microrobots are directly actuated using the magnetic gradient generated by the eight completely independent coils. The differentiation of magnetic forces applied to each microrobot is accomplished by inducing the unique viscous drag on each microrobot when placed in a rotating magnetic field. The different rotational responses result in the different orientations of microrobots, and thence the forces are generated in different directions. Therefore, the proposed EMA system allows different magnetic forces to be exerted on the microrobots, that is capable of enabling the independent remote control and path following of multiple microrobots along arbitrary 3D trajectories. Such electromagnetic microrobotic systems can be used to expand the microrobot techniques for the clinical manipulation, drug delivery, remote sensing and localized therapy in microfluidic channels or in the human body.

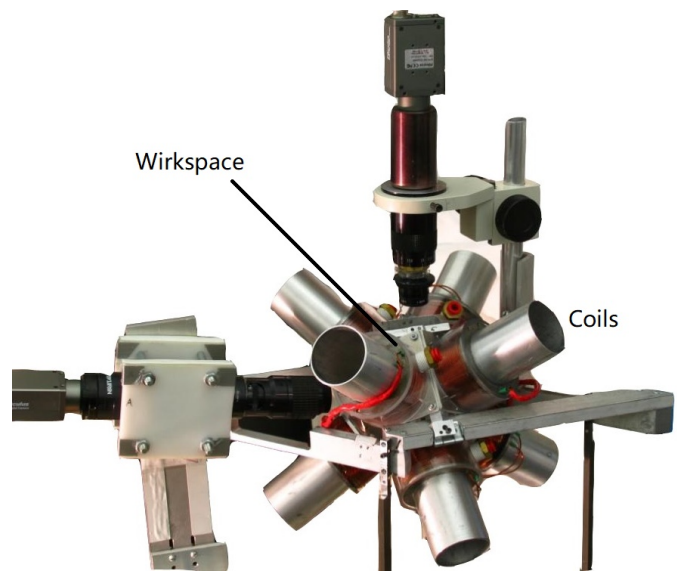

(a)

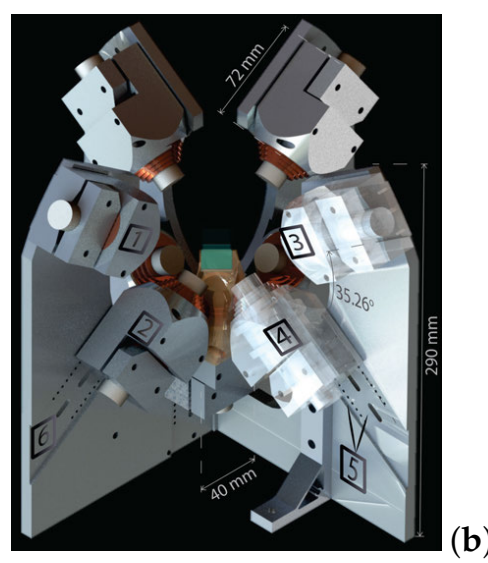

Figure 18. EMA platform developed with independent control: (a) a photograph of the EMA setup comprising 8 electromagnetic coils [95]; and (b) a photograph of the nine-coils EMA system [96].

Indeed, EMA setups with a 3D configuration are more useful for a wider range of biomedical applications than a 2D placement, and other 8 electromagnets configurations such as the well-known OctoMag [59] and MiniMag [60] have been also investigated. The OctoMag platform have been designed with a hemispheric organization of 8 stationary coils. Similarly, the MiniMag system occupies a hemisphere with a tilted arrangement. Compared to OctoMag, the MiniMag setup has been designed to restrict the locations of the electromagnets to a single hemisphere that has a smaller volume. Specifically, they are both developed for the ophthalmic MIS procedure in the retinal veins.

\subsubsection{Platform $9 \mathrm{E}$}

Ongaro et al. [96] have investigated the design of an EMA system composed of nine electromagnetic coils that is capable of independently controlling identical and nonidentical microrobots in the 3D workspace, and named BatMag as illustrated in Figure 18b. The independent control is achieved by exploiting the in-homogeneity of the strong fields that the system can generate. The control strategy for the independent 6 DOFs control of microrobots has been tested and evaluated. Furthermore, a thermal management technique is developed and quantitatively analyzed to prevent overheating during continuous operations. In fact, the Joule heat occurred by the large input current flowing the coil is an important safety factor for EMA setups. This nine-coil EMA system with the independent control could be used for precise collaborative tasks in MIS fields, microassembly, micromanipulation, tissue engineering, and lab-on-a-chip applications. 


\subsection{Analysis}

From the various developed electromagnetic microrobotic setups, the uniform magnetic field can be generated by the Helmholtz coils pair and the uniform saddle coils pair, while the uniform gradient can be produced by the Maxwell coils pair and the gradient saddle coils pair with the different settings, respectively. Such electromagnetic coils form the EMA system with different desired functions. The four stationary coils pairs, such as two pairs of Helmholtz coils and two pairs of Maxwell coils, can control a magnetic microrobot in 2D workspace. The same function of control is also achieved by the four pairs of saddle coils or combination of four coils pairs with the correct configuration. Meanwhile, only two rotating coils pairs can control the 2D motion of microrobots. The one rotating Helmholtz and one rotating Maxwell coils pairs can contribute a 2D position and an orientation control. Similarly, the two rotating saddle coils pairs also can provide the same control with a suitable setting. Thus, all these platforms designed for a 2D workspace allow up to a three DOFs (two translations and one rotation).

When the workspace is expanded to the 3D, at least six stationary independent electromagnetic coils are required. If the coils pair is able to be rotated, the number of coils pair could be reduced. For instance, two pairs of the Helmholtz coils and two pairs of the Maxwell coils can actuate a 3D locomotion of the microrobot where one Helmholtz coils pair and one Maxwell coils pair can rotate along a same axis for rotating a 2D manipulation plane over a 3D workspace. When an electromagnetic coil is used to generate both magnetic field and gradient by adjusting the current, the number of stationary coils pairs can be further reduced to three pairs. In addition, the number and type of coils also depend on the manipulated microrobot. The motion of the microswimmer only requires three pairs of coils that generate the uniform magnetic field throughout the 3D workspace. With the exception of the $3 \mathrm{DH}$ and $9 \mathrm{E}$ platforms, the others allow up to the $5 \mathrm{DOF}$ ( 3 translations and 2 rotations).

The independent manipulation of multiple microrobots has been also investigated $[72,92,95,97]$. It requires either complicated control strategy or special design of the microrobots. Such as, the eight independent electromagnetic coils are responsible for generating the common magnetic gradient to propel the multiple microrobots in the workspace. With the unique viscous drag due to the specific microrobots, their responses are independent to obtain their control. Basically, the electromagnetic actuation system is useful for various manipulation tasks with different configurations of electromagnetic coils under adjustable current inputs and efficient control strategies.

Table 2 summarizes the comparison of the different EMA setups. More comparative and mathematical analyses regarding different configurations of EMA systems have been presented in the previous study [81].

Table 2. Comparisons of the EMA systems.

\begin{tabular}{llll}
\hline EMA & Coils and Workspace & Advantages & Limitations \\
\hline & & $\begin{array}{l}\text { Use few coils with simple } \\
\text { structure; 2 DOFs of } \\
\text { translation and 1 DOF of } \\
\text { rotation; Manipulation of } \\
\text { the orthotropic body. }\end{array}$ & $\begin{array}{l}\text { Low magnetic gradient } \\
\text { over larger areas; } \\
\text { Rotating coils limit } \\
\text { medical applications. }\end{array}$ \\
& 4 coils, 2D & $\begin{array}{l}\text { Less number of coils, 18\% } \\
\text { smaller volume and } \\
\text { 26.7\% less power } \\
\text { consumption to the same } \\
\text { magnetic actuation. }\end{array}$ & $\begin{array}{l}\text { Non-uniform magnetic } \\
\text { field at the edge of } \\
\text { the workspace. }\end{array}$ \\
\hline & 4 coils, 2D & & \\
\hline
\end{tabular}


Table 2. Cont.

\begin{tabular}{|c|c|c|c|}
\hline EMA & Coils and Workspace & Advantages & Limitations \\
\hline 2H2M [65] & Eight coils, 2D & $\begin{array}{l}\text { Simple current control } \\
\text { strategy ; Uniform } \\
\text { magnetic field and } \\
\text { gradient; Manipulation of } \\
\text { the orthotropic body. }\end{array}$ & $\begin{array}{l}\text { Low flexibility of the } \\
\text { confined workspace. }\end{array}$ \\
\hline HMUG [62] & Eight coils, 2D & $\begin{array}{l}\text { Common current control } \\
\text { strategy; Compact setup } \\
\text { with easy access; More } \\
\text { efficient compared to the } \\
\text { Maxwell and Helmholtz } \\
\text { coils; Manipulation of the } \\
\text { orthotropic body. }\end{array}$ & $\begin{array}{l}\text { Size of the proposed } \\
\text { workspace is still limited ; } \\
\text { Non-uniform magnetic } \\
\text { field at the edge of the } \\
\text { workspace; Effective } \\
\text { workspace is limited to } \\
\text { the 2D plane. }\end{array}$ \\
\hline $3 \mathrm{H} 2 \mathrm{M}[90,91]$ & Ten coils, 2D & $\begin{array}{l}\text { Independent control of } \\
\text { the multiple microrobots; } \\
\text { Microparticles with } \\
\text { different shapes. }\end{array}$ & $\begin{array}{l}\text { Only } 2 \text { DOFs motions } \\
\text { (1 DOF rotational and } \\
1 \text { DOF translational) on a } \\
\text { 2D plane; Confined } \\
\text { workspace with difficult } \\
\text { access; Important number } \\
\text { of coils and } \\
\text { power consumption. }\end{array}$ \\
\hline $6 \mathrm{E}[92]$ & Six coils, 2D & $\begin{array}{l}\text { Simultaneous } \\
\text { independent positioning } \\
\text { control of multiple } \\
\text { microrobots; Simple } \\
\text { structure and easy to } \\
\text { implement; } \\
\text { Heterogeneous sets of } \\
\text { dissimilar magnetic } \\
\text { microrobots have been } \\
\text { tested. }\end{array}$ & $\begin{array}{l}\text { Complex control } \\
\text { algorithms, } \\
\text { and limited trajectory. }\end{array}$ \\
\hline 3DMH [74] & Six coils, 3D & $\begin{array}{l}\text { Compact and cheap } \\
\text { setup; 3D manipulation } \\
\text { of the orthotropic body. }\end{array}$ & Limited workspace. \\
\hline 2H2Mr [61] & Eight coils, 3D & $\begin{array}{l}\text { Precise 3D motion; Fewer } \\
\text { number of coils; } \\
\text { Manipulation of the } \\
\text { orthotropic body. }\end{array}$ & $\begin{array}{l}\text { Rotating coils limit } \\
\text { medical applications; } \\
\text { Large setup volume and } \\
\text { power consumption. }\end{array}$ \\
\hline HMUGr [67] & Eight coils, 3D & $\begin{array}{l}\text { Small setup volume and } \\
\text { less consumption; } \\
\text { Workspace accessibility; } \\
\text { Almost all kind of } \\
\text { microrobots. }\end{array}$ & $\begin{array}{l}\text { More powerful current } \\
\text { suppliers are demanded } \\
\text { that will cause } \\
\text { overheating; The } \\
\text { available workspace is } \\
\text { still relatively small. }\end{array}$ \\
\hline $\begin{array}{l}\text { H2US- } \\
\text { MUG [63] }\end{array}$ & Ten coils, 3D & $\begin{array}{l}\text { Compact structure ; } \\
\text { Precise magnetic field } \\
\text { and gradient for 3D } \\
\text { manipulation; Potential } \\
\text { large range of } \\
\text { applications. }\end{array}$ & $\begin{array}{l}\text { Large number of coils; } \\
\text { Small and limited size. }\end{array}$ \\
\hline $3 \mathrm{DH}[94]$ & Six coils, 3D & $\begin{array}{l}6 \text { DOFs motion; Simple } \\
\text { structure and easy to } \\
\text { build; Control of the } \\
\text { helical microswimmer. }\end{array}$ & $\begin{array}{l}\text { Complex algorithm and } \\
\text { control strategy; Only the } \\
\text { magnetic-field-based } \\
\text { control. }\end{array}$ \\
\hline
\end{tabular}


Table 2. Cont.

\begin{tabular}{|c|c|c|c|}
\hline EMA & Coils and Workspace & Advantages & Limitations \\
\hline $8 \mathrm{E}[95]$ & Eight coils, 3D & $\begin{array}{l}\text { Compact structure ; } \\
\text { Arbitrary forces can be } \\
\text { exerted on each } \\
\text { microrobot } \\
\text { independently and } \\
\text { simultaneously. }\end{array}$ & $\begin{array}{l}\text { Small volume with few } \\
\text { accessibility; Weak } \\
\text { magnetic field } \\
\text { and gradients. }\end{array}$ \\
\hline$[59,60]$ & & $\begin{array}{l}\text { Good magnetic field; } \\
\text { Hemispherical coils } \\
\text { arrangement. }\end{array}$ & $\begin{array}{l}\text { Complex magnetic } \\
\text { field description. }\end{array}$ \\
\hline $9 \mathrm{E}[96]$ & Nine coils, 3D & $\begin{array}{l}\text { Independent 3D control } \\
\text { of pairs of microrobots; } 6 \\
\text { DOFs motion; Workspace } \\
\text { accessibility. }\end{array}$ & $\begin{array}{l}\text { Advanced control } \\
\text { strategies are required; } \\
\text { Large amount of energy } \\
\text { consumption } \\
\text { and heating. }\end{array}$ \\
\hline
\end{tabular}

\section{Conclusions}

We have provided a comprehensive review of the current advances of the biomedical microrobot in MIS and a comparison of various magnetic microrobotic systems developed by many researchers. One of the highest potential scientific and societal impact of the untethered microrobot would be the health-care and bio-engineering applications. As the robot size is decreased to the micro or nano-scale, previously inaccessible body locations would become available for high-resolution in situ and in vivo operations. Such remotely access will enable an extensive range of MIS operations. To this aim, the untethered microrobot would be wirelessly manipulated by the magnetic torque and force induced thanks to an EMA system. To precisely manipulate the biomedical microrobot, a key issue still relies on the suitable configuration of EMA platforms.

Therefore, this work is devoted to present a general review of magnetic microrobots and magnetic microrobotic actuation setups. Especially, the choice of suitable magnetic microrobotic platform is a significant issue for the desired biomedical applications. The magnetic microrobotic systems with different configurations can produce respectively the magnetic field and gradient. As illustrated in Figure 1A, the magnetic field and gradient on the 2D surface generated by flat-arrangement magnetic microrobotic system are appropriate for the surface or skin operations. For the deeper surgical treatment, the magnetic microrobotic system can be utilized to control the medical microrobot to operate on members (e.g., arms, legs) for targeted therapy. This allows increasing the concentration of drugs in the part to be treated and reduces the impact on other parts of the body, that makes the treatment more effective and minimize side effects. Thus, the magnetic microrobotic system could be designed to surround the desired workspace as represented in Figure 1B since the applied part could be placed through the setup. Once again, with the requirements of a specific intraocular application, since the magnetic field with a safe intensity is arduous to launch deep into the body tissue providing enough magnetic field and gradient, the coils generating the magnetic field placed behind the head would be futile for the eye operation. Hence, the applied coils are all arranged around the eye in the front of the head. It is clear that the various magnetic microrobotic systems are utilized specifically for the pertinent biomedical applications in order to perform efficiently their functions.

Author Contributions: Methodology, R.C.; Analysis, R.C. and D.F.; Writing-original draft, R.C. and D.F.; Writing—review and editing, R.C., D.F. and A.F.; Project administration, A.F.; Funding Acquisition, A.F. All authors have read and agreed to the published version of the manuscript.

Funding: This work was supported by the French National Institute of Health and Medical Research (Inserm) "Plan Cancer" 2014-2019 program, Project: Microrobots Targeting Glioblastoma (MTG). 
Institutional Review Board Statement: Not applicable.

Informed Consent Statement: The data presented in this study are available from the corresponding authors upon reasonable request.

Data Availability Statement: Not applicable.

Acknowledgments: This work was supported by French National Institute of Health and Medical Research (Inserm) "Plan Cancer" program and the Microrobots Targeting Glioblastoma (MTG) project.

Conflicts of Interest: The authors declare no conflict of interest.

\author{
Abbreviations \\ DOF Degree of Freedom \\ EMA Electromagnetic Actuation \\ MIS Minimally Invasive Surgery
}

The following abbreviations are used in this manuscript:

\title{
References
}

1. Tendick, F.; Sastry, S.S.; Fearing, R.S.; Cohn, M. Applications of micromechatronics in minimally invasive surgery. IEEE/ASME Trans. Mechatron. 1998, 3, 34-42. [CrossRef]

2. Mack, M.J. Minimally invasive and robotic surgery. JAMA 2001, 285, 568-572. [CrossRef]

3. Carrozza, M.C.; Dario, P.; Jay, L.P.S. Micromechatronics in surgery. Trans. Inst. Meas. Control 2003, 25, 309-327. [CrossRef]

4. Purkayastha, S.; Athanasiou, T.; Casula, R.; Darzi, S.A. Robotic surgery: A review. Hosp. Med. 2004, 65, 153-159. [CrossRef]

5. Joseph, J.V.; Arya, M.; Patel, H.R. Robotic surgery: The coming of a new era in surgical innovation. Expert Rev. Anticancer Ther. 2005, 5, 7-9. [CrossRef] [PubMed]

6. Bogue, R. The development of medical microrobots: A review of progress. Ind. Robot. Int. J. 2008, 35, 294-299. [CrossRef]

7. Broeders, I.A.; Ruurda, J. Robotics revolutionizing surgery: The intuitive surgical "da Vinci" system. Ind. Robot. Int. J. 2001, 28, 387-392. [CrossRef]

8. Tsuda, S.; Oleynikov, D.; Gould, J.; Azagury, D.; Sandler, B.; Hutter, M.; Ross, S.; Haas, E.; Brody, F.; Satava, R. SAGES TAVAC safety and effectiveness analysis: Da Vinci ${ }^{\circledR}$ surgical system (Intuitive Surgical, Sunnyvale, CA). Surg. Endosc. 2015, 29, $2873-2884$. [CrossRef] [PubMed]

9. Baek, S.J.; Kim, S.H. Robotics in general surgery: An evidence-based review. Asian J. Endosc. Surg. 2014, 7, 117-123. [CrossRef]

10. Grimsby, G.M.; Dwyer, M.E.; Jacobs, M.A.; Ost, M.C.; Schneck, F.X.; Cannon, G.M.; Gargollo, P.C. Multi-institutional review of outcomes of robot-assisted laparoscopic extravesical ureteral reimplantation. J. Urol. 2015, 193, 1791-1795. [CrossRef] [PubMed]

11. Morelli, L.; Guadagni, S.; Di Franco, G.; Palmeri, M.; Di Candio, G.; Mosca, F. Da Vinci single site $\bigodot$ surgical platform in clinical practice: A systematic review. Int. J. Med Robot. Comput. Assist. Surg. 2016, 12, 724-734. [CrossRef]

12. Ferreira, A. (Ed.) The Encyclopedia of Medical Robotics; World Scientific: Singapore, 2018; doi: 10.1142/10770. [CrossRef]

13. Yang, G.Z.; Bellingham, J.; Dupont, P.E.; Fischer, P.; Floridi, L.; Full, R.; Jacobstein, N.; Kumar, V.; McNutt, M.; Merrifield, R.; et al. The grand challenges of Science Robotics. Sci. Robot. 2018, 3, eaar7650. [CrossRef] [PubMed]

14. Simaan, N.; Yasin, R.M.; Wang, L. Medical technologies and challenges of robot-assisted minimally invasive intervention and diagnostics. Annu. Rev. Control. Robot. Auton. Syst. 2018, 1, 465-490. [CrossRef]

15. Rattner, D.; Kalloo, A. ASGE/SAGES working group on natural orifice translumenal endoscopic surgery. Surg. Endosc. Other Interv. Tech. 2006, 20, 329-333. [CrossRef] [PubMed]

16. Romanelli, J.R.; Earle, D.B. Single-port laparoscopic surgery: An overview. Surg. Endosc. 2009, 23, 1419-1427. [CrossRef] [PubMed]

17. Tiwari, M.M.; Reynoso, J.F.; Lehman, A.C.; Tsang, A.W.; Farritor, S.M.; Oleynikov, D. In vivo miniature robots for natural orifice surgery: State of the art and future perspectives. World J. Gastrointest. Surg. 2010, 2, 217. [CrossRef]

18. Samarasekera, D.; Kaouk, J.H. Robotic single port surgery: Current status and future considerations. Indian J. Urol. IJU J. Urol. Soc. India 2014, 30, 326.

19. Bae, S.U.; Jeong, W.K.; Baek, S.K. Current status of robotic single-port colonic surgery. Int. J. Med Robot. Comput. Assist. Surg. 2017, 13, e1735. [CrossRef]

20. Courreges, F.; Vieyres, P.; Poisson, G. Robotized tele-echography. In Teleradiology; Springer International Publishing: Berlin/Heidelberg, Germany, 2008; pp. 139-154.

21. Sitti, M. Miniature devices: Voyage of the microrobots. Nature 2009, 458, 1121. [CrossRef]

22. Nelson, B.J.; Kaliakatsos, I.K.; Abbott, J.J. Microrobots for minimally invasive medicine. Annu. Rev. Biomed. Eng. 2010, 12, 55-85. [CrossRef] [PubMed]

23. Ceylan, H.; Giltinan, J.; Kozielski, K.; Sitti, M. Mobile microrobots for bioengineering applications. Lab Chip 2017, 17, 1705-1724. [CrossRef] 
24. Honda, T.; Arai, K.; Ishiyama, K. Micro swimming mechanisms propelled by external magnetic fields. IEEE Trans. Magn. 1996, 32, 5085-5087. [CrossRef]

25. Abbott, J.J.; Peyer, K.E.; Lagomarsino, M.C.; Zhang, L.; Dong, L.; Kaliakatsos, I.K.; Nelson, B.J. How should microrobots swim? Int. J. Robot. Res. 2009, 28, 1434-1447. [CrossRef]

26. Alapan, Y.; Yasa, O.; Schauer, O.; Giltinan, J.; Tabak, A.F.; Sourjik, V.; Sitti, M. Soft erythrocyte-based bacterial microswimmers for cargo delivery. Sci. Robot. 2018, 3, eaar4423. [CrossRef]

27. Barbot, A.; Decanini, D.; Hwang, G. The Rotation of Microrobot Simplifies 3D Control Inside Microchannels. Sci. Rep. 2018, 8, 438. [CrossRef]

28. Carlsen, R.W.; Edwards, M.R.; Zhuang, J.; Pacoret, C.; Sitti, M. Magnetic steering control of multi-cellular bio-hybrid microswimmers. Lab Chip 2014, 14, 3850-3859. [CrossRef] [PubMed]

29. Carpi, F.; Kastelein, N.; Talcott, M.; Pappone, C. Magnetically controllable gastrointestinal steering of video capsules. IEEE Trans. Biomed. Eng. 2011, 58, 231-234. [CrossRef]

30. Diller, E.; Sitti, M. Micro-scale mobile robotics. Found. Trends® Robot. 2013, 2, 143-259. [CrossRef]

31. Ghosh, A.; Fischer, P. Controlled propulsion of artificial magnetic nanostructured propellers. Nano Lett. 2009, 9, $2243-2245$. [CrossRef] [PubMed]

32. Hosney, A.; Abdalla, J.; Amin, I.S.; Hamdi, N.; Khalil, I.S. In vitro validation of clearing clogged vessels using microrobots. In Proceedings of the IEEE International Conference on Biomedical Robotics and Biomechatronics (BioRob), Singapore, 26-29 June 2016; pp. 272-277.

33. Jeon, S.; Kim, S.; Ha, S.; Lee, S.; Kim, E.; Kim, S.Y.; Park, S.H.; Jeon, J.H.; Kim, S.W.; Moon, C.; et al. Magnetically actuated microrobots as a platform for stem cell transplantation. Sci. Robot. 2019, 4, eaav4317. [CrossRef]

34. Kim, S.; Qiu, F.; Kim, S.; Ghanbari, A.; Moon, C.; Zhang, L.; Nelson, B.J.; Choi, H. Fabrication and characterization of magnetic microrobots for three-dimensional cell culture and targeted transportation. Adv. Mater. 2013, 25, 5863-5868. [CrossRef]

35. Du Nguyen, V.; Le, V.H.; Zheng, S.; Han, J.; Park, J.O. Preparation of tumor targeting cell-based microrobots carrying NIR light sensitive therapeutics manipulated by electromagnetic actuating system and Chemotaxis. J. Micro-Bio Robot. 2018, 14, 69-77. [CrossRef]

36. Lee, S.; Lee, S.; Kim, S.; Yoon, C.H.; Park, H.J.; Kim, J.y.; Choi, H. Fabrication and characterization of a magnetic drilling actuator for navigation in a three-dimensional phantom vascular network. Sci. Rep. 2018, 8, 3691. [CrossRef]

37. Li, J.; de Ávila, B.E.F.; Gao, W.; Zhang, L.; Wang, J. Micro/nanorobots for biomedicine: Delivery, surgery, sensing, and detoxification. Sci. Robot. 2017, 2, 18-23. [CrossRef] [PubMed]

38. Li, J.; Li, X.; Luo, T.; Wang, R.; Liu, C.; Chen, S.; Li, D.; Yue, J.; Cheng, S.H.; Sun, D. Development of a magnetic microrobot for carrying and delivering targeted cells. Sci. Robot 2018, 3, eaat8829. [CrossRef]

39. Martel, S. Microrobotics in the vascular network: Present status and next challenges. J. Micro-Bio Robot. 2013, 8, 41-52. [CrossRef]

40. Mitchell, B.; Koo, J.; Iordachita, I.; Kazanzides, P.; Kapoor, A.; Handa, J.; Hager, G.; Taylor, R. Development and application of a new steady-hand manipulator for retinal surgery. In Proceedings of the IEEE International Conference on Robotics and Automation (ICRA), Roma, Italy, 10-14 April 2007; pp. 623-629.

41. Palagi, S.; Fischer, P. Bioinspired microrobots. Nat. Rev. Mater. 2018, 3, 113-124. [CrossRef]

42. Park, S.; Cha, K.; Park, J. Development of biomedical microrobot for intravascular therapy. Int. J. Adv. Robot. Syst. 2010, 7, 91-98. [CrossRef]

43. Peyer, K.E.; Zhang, L.; Nelson, B.J. Bio-inspired magnetic swimming microrobots for biomedical applications. Nanoscale 2013, 5, 1259-1272. [CrossRef] [PubMed]

44. Qiu, F.; Nelson, B.J. Magnetic helical micro-and nanorobots: Toward their biomedical applications. Engineering 2015, 1, 21-26. [CrossRef]

45. Schürle, S.; Kratochvil, B.E.; Pané, S.; Zeeshan, M.A.; Nelson, B.J. Generating Magnetic Fields for Controlling Nanorobots in Medical Applications. In Nanorobotics; Springer International Publishing: Berlin/Heidelberg, Germany, 2013; pp. 275-299.

46. Sitti, M.; Ceylan, H.; Hu, W.; Giltinan, J.; Turan, M.; Yim, S.; Diller, E.D. Biomedical Applications of Untethered Mobile Milli/Microrobots. Proc. IEEE 2015, 103, 205-224. [CrossRef]

47. Xie, H.; Sun, M.; Fan, X.; Lin, Z.; Chen, W.; Wang, L.; Dong, L.; He, Q. Reconfigurable magnetic microrobot swarm: Multimode transformation, locomotion, and manipulation. Sci. Robot. 2019, 4, eaav8006. [CrossRef]

48. Yesin, K.B.; Exner, P.; Vollmers, K.; Nelson, B.J. Design and control of in vivo magnetic microrobots. In Proceedings of the International Conference on Medical Image Computing and Computer-Assisted Intervention, Palm Springs, CA, USA, 26-29 October 2005; Springer International Publishing: Berlin/Heidelberg, Germany, 2005; pp. 819-826.

49. Yesin, K.B.; Vollmers, K.; Nelson, B.J. Modeling and control of untethered biomicrorobots in a fluidic environment using electromagnetic fields. Int. J. Robot. Res. 2006, 25, 527-536. [CrossRef]

50. Steager, E.B.; Selman Sakar, M.; Magee, C.; Kennedy, M.; Cowley, A.; Kumar, V. Automated biomanipulation of single cells using magnetic microrobots. Int. J. Robot. Res. 2013, 32, 346-359. [CrossRef]

51. Fountain, T.W.; Kailat, P.V.; Abbott, J.J. Wireless control of magnetic helical microrobots using a rotating-permanent-magnet manipulator. In Proceedings of the IEEE International Conference on Robotics and Automation (ICRA), Anchorage, AK, USA, 3-7 May 2010; pp. 576-581. 
52. Torres, N.A.; Ruggeri, S.; Popa, D.O. Untethered microrobots actuated with focused permanent magnet field. In Proceedings of the ASME 2014 International Design Engineering Technical Conferences and Computers and Information in Engineering Conference, Buffalo, NY, USA, 17 August 2014; p. V004T09A024.

53. Mahoney, A.W.; Abbott, J.J. Five-degree-of-freedom manipulation of an untethered magnetic device in fluid using a single permanent magnet with application in stomach capsule endoscopy. Int. J. Robot. Res. 2016, 35, 129-147. [CrossRef]

54. Lucarini, G.; Ciuti, G.; Mura, M.; Rizzo, R.; Menciassi, A. A new concept for magnetic capsule colonoscopy based on an electromagnetic system. Int. J. Adv. Robot. Syst. 2015, 12, 25. [CrossRef]

55. Ryan, P.; Diller, E. Magnetic actuation for full dexterity microrobotic control using rotating permanent magnets. IEEE Trans. Robot. 2017, 33, 1398-1409. [CrossRef]

56. Amokrane, W.; Belharet, K.; Souissi, M.; Grayeli, A.B.; Ferreira, A. Macro-micromanipulation platform for inner ear drug delivery. Robot. Auton. Syst. 2018, 107, 10-19. [CrossRef]

57. Schuerle, S.; Erni, S.; Flink, M.; Kratochvil, B.E.; Nelson, B.J. Three-dimensional magnetic manipulation of micro-and nanostructures for applications in life sciences. IEEE Trans. Magn. 2013, 49, 321-330. [CrossRef]

58. Martel, S.; Mathieu, J.B.; Felfoul, O.; Chanu, A.; Aboussouan, E.; Tamaz, S.; Pouponneau, P.; Yahia, L.; Beaudoin, G.; Soulez, G.; et al. Automatic navigation of an untethered device in the artery of a living animal using a conventional clinical magnetic resonance imaging system. Appl. Phys. Lett. 2007, 90, 114105. [CrossRef]

59. Kummer, M.P.; Abbott, J.J.; Kratochvil, B.E.; Borer, R.; Sengul, A.; Nelson, B.J. OctoMag: An electromagnetic system for 5-DOF wireless micromanipulation. IEEE Trans. Robot. 2010, 26, 1006-1017. [CrossRef]

60. Kratochvil, B.E.; Kummer, M.P.; Erni, S.; Borer, R.; Frutiger, D.R.; Schürle, S.; Nelson, B.J. MiniMag: A hemispherical electromagnetic system for 5-DOF wireless micromanipulation. In Experimental Robotics; Springer International Publishing: Berlin/Heidelberg, Germany, 2014; pp. 317-329.

61. Jeong, S.; Choi, H.; Choi, J.; Yu, C.; Park, J.O.; Park, S. Novel electromagnetic actuation (EMA) method for 3-dimensional locomotion of intravascular microrobot. Sens. Actuators A Phys. 2010, 157, 118-125. [CrossRef]

62. Jeon, S.; Jang, G.; Choi, H.; Park, S. Magnetic navigation system with gradient and uniform saddle coils for the wireless manipulation of micro-robots in human blood vessels. IEEE Trans. Magn. 2010, 46, 1943-1946. [CrossRef]

63. Jeon, S.; Jang, G.; Choi, H.; Park, S.; Park, J. Magnetic navigation system for the precise helical and translational motions of a microrobot in human blood vessels. J. Appl. Phys. 2012, 111, 07E702. [CrossRef]

64. Choi, H.; Choi, J.; Jeong, S.; Yu, C.; Park, J.O.; Park, S. Two-dimensional locomotion of a microrobot with a novel stationary electromagnetic actuation system. Smart Mater. Struct. 2009, 18, 115017. [CrossRef]

65. Choi, H.; Choi, J.; Jang, G.; Park, J.O.; Park, S. Two-dimensional actuation of a microrobot with a stationary two-pair coil system. Smart Mater. Struct. 2009, 18, 055007. [CrossRef]

66. Choi, J.; Jeong, S.; Cha, K.; Qin, L.; Li, J.; Park, J.; Park, S. Positioning of microrobot in a pulsating flow using EMA system. In Proceedings of the IEEE RAS \& EMBS International Conference on Biomedical Robotics and Biomechatronics, Tokyo, Japan, 26-29 September 2010; pp. 588-593.

67. Choi, H.; Cha, K.; Choi, J.; Jeong, S.; Jeon, S.; Jang, G.; Park, J.O.; Park, S. EMA system with gradient and uniform saddle coils for 3D locomotion of microrobot. Sens. Actuators A Phys. 2010, 163, 410-417. [CrossRef]

68. Choi, H.; Cha, K.; Jeong, S.; Park, J.O.; Park, S. 3-D locomotive and drilling microrobot using novel stationary EMA system. IEEE/ASME Trans. Mechatron. 2012, 18, 1221-1225. [CrossRef]

69. Choi, H.; Park, J.O.; Park, S. Electromagnetic Actuated Micro- and Nanorobots; World Scientific: Singapore, 2018; Volume 2, Chapter 6, pp. 113-135. [CrossRef]

70. Go, G.; Choi, H.; Jeong, S.; Lee, C.; Park, B.J.; Ko, S.Y.; Park, J.O.; Park, S. Position-based magnetic field control for an electromagnetic actuated microrobot system. Sens. Actuators A Phys. 2014, 205, 215-223. [CrossRef]

71. Go, G.; Choi, H.; Jeong, S.; Lee, C.; Ko, S.Y.; Park, J.O.; Park, S. Electromagnetic navigation system using simple coil structure (4 coils) for 3-D locomotive microrobot. IEEE Trans. Magn. 2015, 51, 1-7.

72. Diller, E.; Giltinan, J.; Jena, P.; Sitti, M. Three dimensional independent control of multiple magnetic microrobots. In Proceedings of the IEEE International Conference on Robotics and Automation (ICRA), Karlsruhe, Germany, 6-10 May 2013; pp. $2576-2581$.

73. Diller, E.; Giltinan, J.; Lum, G.Z.; Ye, Z.; Sitti, M. Six-degree-of-freedom magnetic actuation for wireless microrobotics. Int. J. Robot. Res. 2016, 35, 114-128. [CrossRef]

74. Dadkhah, M.; Kumar, N.; Yoon, J. Design and simulation of a 3D actuation system for magnetic nano-particles delivery system. In Proceedings of the International Conference on Intelligent Robotics and Applications, Busan, Korea, 25-28 September 2013; Springer International Publishing: Berlin/Heidelberg, Germany, 2013; pp. 177-187.

75. Fu, Q.; Guo, S.; Huang, Q.; Hirata, H.; Ishihara, H. Development and evaluation of novel magnetic actuated microrobot with spiral motion using electromagnetic actuation system. J. Med Biol. Eng. 2016, 36, 506-514. [CrossRef]

76. Tehrani, M.D.; Kim, M.O.; Yoon, J. A novel electromagnetic actuation system for magnetic nanoparticle guidance in blood vessels. IEEE Trans. Magn. 2014, 50, 1-12. [CrossRef]

77. Yu, C.; Kim, J.; Choi, H.; Choi, J.; Jeong, S.; Cha, K.; Park, J.O.; Park, S. Novel electromagnetic actuation system for threedimensional locomotion and drilling of intravascular microrobot. Sens. Actuators A Phys. 2010, 161, 297-304. [CrossRef]

78. Abbott, J.J.; Diller, E.; Petruska, A.J. Magnetic Methods in Robotics. Annu. Rev. Control. Robot. Auton. Syst. 2015, 3, 57-90. [CrossRef] 
79. Charreyron, S.L.; Boehler, Q.; Kim, B.; Weibel, C.; Chautems, C.; Nelson, B.J. Modeling Electromagnetic Navigation Systems. IEEE Trans. Robot. 2021, 37, 1009-1021. [CrossRef]

80. Yu, R.; Charreyron, S.L.; Boehler, Q.; Weibel, C.; Chautems, C.; Poon, C.C.; Nelson, B.J. Modeling Electromagnetic Navigation Systems for Medical Applications using Random Forests and Artificial Neural Networks. In Proceedings of the 2020 IEEE International Conference on Robotics and Automation (ICRA), Virtual Conference, 1 June-31 August 2020; pp. 9251-9256.

81. Chen, R.; Folio, D.; Ferreira, A. Mathematical approach for the design configuration of magnetic system with multiple electromagnets. Robot. Auton. Syst. 2021, 135, 103674. [CrossRef]

82. Miyashita, S.; Guitron, S.; Yoshida, K.; Li, S.; Damian, D.D.; Rus, D. Ingestible, controllable, and degradable origami robot for patching stomach wounds. In Proceedings of the IEEE International Conference on Robotics and Automation (ICRA), Stockholm, Sweden, 16-21 May 2016; pp. 909-916.

83. Petruska, A.J.; Nelson, B.J. Minimum bounds on the number of electromagnets required for remote magnetic manipulation. IEEE Trans. Robot. 2015, 31, 714-722. [CrossRef]

84. Ko, Y.; Na, S.; Lee, Y.; Cha, K.; Ko, S.Y.; Park, J.; Park, S. A jellyfish-like swimming mini-robot actuated by an electromagnetic actuation system. Smart Mater. Struct. 2012, 21, 057001. [CrossRef]

85. Mathieu, J.B.; Martel, S.; Yahia, L.; Soulez, G.; Beaudoin, G. Preliminary studies for using magnetic resonance imaging systems as a mean of propulsion for microrobots in blood vessels and evaluation of ferromagnetic artefacts. In Proceedings of the Canadian Conference on Electrical and Computer Engineering. Toward a Caring and Humane Technology, Montreal, QC, Canada, 4-7 May 2003; Volume 2, pp. 835-838.

86. Martel, S.; Mathieu, J.B.; Felfoul, O.; Macicior, H.; Beaudoin, G.; Soulez, G.; Yahia, L. Adapting MRI systems to propel and guide microdevices in the human blood circulatory system. In Proceedings of the Annual International Conference of the IEEE Engineering in Medicine and Biology Society, San Francisco, CA, USA, 1-5 September 2004; Volume 1, pp. $1044-1047$.

87. Bigot, A.; Tremblay, C.; Soulez, G.; Martel, S. Magnetic resonance navigation of a bead inside a three-bifurcation pmma phantom using an imaging gradient coil insert. IEEE Trans. Robot. 2014, 30, 719-727. [CrossRef]

88. Véron, B.; Hubert, A.; Abadie, J.; Andreff, N. Geometric analysis of the singularities of a magnetic manipulation system with several mobile coils. In Proceedings of the IEEE/RSJ International Conference on Intelligent Robots and Systems (IROS), Chicago, IL, USA, 14-18 September 2013; pp. 4996-5001.

89. Yang, L.; Du, X.; Yu, E.; Jin, D.; Zhang, L. DeltaMag: An Electromagnetic Manipulation System with Parallel Mobile Coils. In Proceedings of the International Conference on Robotics and Automation (ICRA), Montreal, QC, Canada, 20-24 May 2019; pp. 9814-9820.

90. Go, G.; Choi, H.; Lee, C.; Ko, S.Y.; Park, J.O.; Park, S. A multiple microrobot system with thermally responsive microclampers. In Proceedings of the IEEE International Conference on Biomedical Robotics and Biomechatronics (BioRob), Twente, The Netherlands, 24-26 August 2016; pp. 1186-1191.

91. Go, G.; Choi, H.; Jeong, S.; Ko, S.Y.; Park, J.O.; Park, S. Selective microrobot control using a thermally responsive microclamper for microparticle manipulation. Smart Mater. Struct. 2016, 25, 035004. [CrossRef]

92. Diller, E.; Floyd, S.; Pawashe, C.; Sitti, M. Control of multiple heterogeneous magnetic microrobots in two dimensions on nonspecialized surfaces. IEEE Trans. Robot. 2011, 28, 172-182. [CrossRef]

93. Tamaz, S.; Gourdeau, R.; Chanu, A.; Mathieu, J.B.; Martel, S. Real-time MRI-based control of a ferromagnetic core for endovascular navigation. IEEE Trans. Biomed. Eng. 2008, 55, 1854-1863. [CrossRef] [PubMed]

94. Mahoney, A.W.; Sarrazin, J.C.; Bamberg, E.; Abbott, J.J. Velocity control with gravity compensation for magnetic helical microswimmers. Adv. Robot. 2011, 25, 1007-1028. [CrossRef]

95. Diller, E.; Giltinan, J.; Sitti, M. Independent control of multiple magnetic microrobots in three dimensions. Int. J. Robot. Res. 2013, 32, 614-631. [CrossRef]

96. Ongaro, F.; Pane, S.; Scheggi, S.; Misra, S. Design of an electromagnetic setup for independent three-dimensional control of pairs of identical and nonidentical microrobots. IEEE Trans. Robot. 2018, 35, 174-183. [CrossRef]

97. Floyd, S.; Pawashe, C.; Sitti, M. Microparticle manipulation using multiple untethered magnetic micro-robots on an electrostatic surface. In Proceedings of the IEEE/RSJ International Conference on Intelligent Robots and Systems (IROS), St. Louis, MO, USA, 10-15 October 2009; pp. 528-533. 